\title{
A Study on Optimal Planning Strategy Based on Customer Outage Costs
}

\begin{abstract}
Balho H. Kim ${ }^{\dagger}$
Abstract - This paper examines the long-run social welfare maximization problems facing public utilities, which includes consideration of the cost of capital or other fixed costs of production, from which it derives optimal investment decisions in a reliability differentiated pricing based market. Reliability differentiated pricing policies lead to straightforward mathematical results on optimal investment decisions in generation and transmission expansion planning. This paper presents the mathematical conditions for optimal investment decisions.
\end{abstract}

Keywords: Reliability differentiated pricing, Optimal investment planning, Social welfare maximization, Long-run system expansion planning

\section{Introduction}

Outage costs represent the economic and non-economic consequences of service curtailments to the customer [1], [2]. These are traditionally estimated by the customer's willingness-to-pay (WTP) to avoid interruptions in the short-run, assuming the user does not have sufficient time to change his energy using capital stock [3].

This varies between customers within a particular class, and between classes of customers in general. The variation will be a function of the degree to which customer applications are dependent upon electricity and to what extent any production or service can be recovered subsequent to an outage.

The long-run problem of a welfare maximizing utility is essentially a resource planning problem using the criterion of maximizing expected consumers' plus producers' surplus subject to the operational and resource limits of the system [4], [5]. Financial constraints on the utility or any of the participants, which is particularly important for developing countries, will be ignored in the analysis to follow. It is assumed that a single welfare maximizing public utility owns and operates the transmission network (and possibly the generating plants) of the electric power system under consideration and it sells to independent customers. The utility is assumed to be able to set and communicate prices instantly, and can set a different price for each customer class at any particular time. Supply outages are assumed to occur randomly with the probability of an outage possibly being known. Thus, customers are aware of an outage only after it has occurred. The utility is assumed to be able to ration supply shortages amongst the various customer classes as it chooses. In the analysis to follow, both generator outages and transmission line outages will be considered.

$\dagger$ Corresponding Author: School of Electrical Engineering at the Hongik University, Korea.( bhkim@wow.hongik.ac.kr)

Received : November 7, 2008; Accepted : December 28, 2009

\section{The Long-run Model}

\subsection{Objective Functions}

\subsubsection{Consumer Behavior [5]}

Customers are modeled as price-taking expected profitmaximizing firms [6]. Assume that all customers of the system, or more accurately customer end users, can be classified into a finite number of classes, $i \in I, I=\{1, \ldots, N\}$, each class consisting of customers having the same outage cost at a particular location. The total time period, T, is considered to consist of short discrete time intervals $\mathrm{t} \in \mathrm{T}$.

Consider the case of a long-term rational customer $\mathrm{i}$ whose benefit at time t, $F_{i t}=F_{i t}\left(P^{d}(p), Q^{d}(q)\right)$, depends not only on their electricity usage at that time but also on usage at other times and even possibly on usage by other customers (end users). i.e.

$$
F_{i t}=F_{i t}\left(P^{d}(p), Q^{d}(q)\right)
$$

Where,

$$
\begin{aligned}
& P^{d}=\left\{P_{i t}^{d} ; \forall i \in I, \forall t \in T\right\} \\
& : \text { vector of real power demands, } \\
& Q^{d}=\left\{Q_{i t}^{d} ; \forall i \in I, \forall t \in T\right\} \\
& : \text { vector of reactive power demands, } \\
& \mathrm{p}: \text { vector of prices of real power, } \\
& \mathrm{q}: \text { vector of prices of reactive power. }
\end{aligned}
$$

Assume the customer seeks to maximize their total ex-

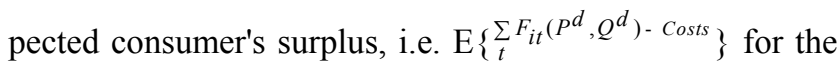
time period $\mathrm{T}$, where $\mathrm{E}$ is the expectated operator. Thus, the customer will choose ${ }_{i t}^{P_{i t}^{d}, Q_{i t}^{d}}$ to maximize their expected profit: 


$$
\begin{aligned}
& M a x: \pi=E\left[\sum _ { t } \left\{F_{i t}\left(P^{d}, Q^{d}\right)\right.\right. \\
& \left.\left.-O C_{i t}^{d}\left(P^{d}, Q^{d}\right)-P_{i t} P_{i t}^{d}-{ }_{q_{i t}} Q_{i t}^{d}\right\}\right] \\
\Rightarrow \quad & \rightarrow \frac{\partial \sum_{\tau \in T} F_{i t}}{\partial P_{i t}^{d}}-\frac{\partial \sum_{\tau \in T} O C_{i t}}{\partial P_{i t}^{d}}=P_{i t} \\
\quad \rightarrow & \frac{\partial \sum_{\tau \in T} F_{i t}}{\partial Q_{i t}^{d}}-\frac{\partial \sum_{\tau \in T} O C_{i t}}{\partial Q_{i t}^{d}}=q_{i t}
\end{aligned}
$$

where $P_{i t}, q_{i t}$ are the expected prices of real and reactive power, respectively, $O C_{i t}^{d}\left(P^{d}, Q^{d}\right)$ is the outage cost of the customer as a function of demand. Thus, the customer equates their expected net marginal benefit to the expected price.

\subsubsection{Utility Behavior}

The objective of the long-run resource planning problem for a welfare maximizing utility using the criterion of maximizing expected consumers' plus producers' surplus can be stated as follows [7], [8]:

$$
\begin{aligned}
\text { Max } & : W=E\left[\sum _ { t } \left\{\sum_{i} F_{i t}\left(P_{i}^{d}, Q_{i}^{d}\right)\right.\right. \\
& -\sum_{i} O C_{i t}\left(P_{i}^{u}, Q_{i}^{u}\right)-\sum_{i} P C_{i t}\left(P_{i t}^{g}, Q_{i t}^{g}\right) \\
& \left.\left.-\sum_{i} E C_{i t}\left(P_{i t}^{s}, Q_{i t}^{s}\right)\right\}\right]-\sum_{i} C C_{i}\left(K_{i}^{p}, K_{i}^{q}\right) \\
& -\sum_{i} S C_{i}\left(S_{i}^{p}, S_{i}^{q}\right)-\sum_{K} T C_{k}\left(T_{k}^{\max }\right)
\end{aligned}
$$

where,

$P_{i}{ }^{d} \quad$ : real power demand of customer $\mathrm{i}$

$Q_{i}^{d} \quad$ : reactive power demand of customer $\mathrm{i}$

$P_{i}^{u} \quad$ : outage of real power for customer i

$Q_{i}^{u} \quad$ : outage of reactive power for customer i,

$P_{i}^{g} \quad$ : generation of real power by generator i

$Q_{i}^{g} \quad$ : generation of reactive power by generator i,

$P_{i}{ }^{s} \quad$ : emergency purchase of real power at bus $\mathrm{i}$

$Q_{i}^{s} \quad$ : emergency purchase of reactive power at bus i,

$K_{i}^{p} \quad$ : real power generation capacity of generator I,

$K_{i}{ }^{q} \quad$ : reactive power generation capacity of generator I,

$S_{i}^{p} \quad$ : real power spinning reserve capacity at bus i

$S_{i}^{q} \quad$ : reactive power spinning reserve capacity at bus i

$T_{k}{ }^{\text {max }}$ : volt-ampere transmission capacity of line $\mathrm{k}$

are the decision variables, and

$\mathrm{E}[\bullet] \quad:$ the expected value of the argument,

$F_{i t}\left(P_{i}^{d}, Q_{i}^{d}\right) \quad$ : value-added function of customer i,

$O C_{i t}\left(P_{i}{ }^{u}, Q_{i}^{u}\right) \quad$ : outage cost function of customer i,

$P C_{i t}\left(P_{i t}^{g}, Q_{i t}^{g}\right) \quad:$ cost of producing real and reactive power by unit $\mathrm{i}$,

$E C_{i t}\left(P_{i t}^{s}, Q_{i t}^{s}\right)$ : cost of purchasing real and reactive power by unit i,

$C C_{i}\left(K_{i}^{p}, K_{i}^{q}\right)$ : capital cost of capacity for generator i,

$S C_{i}\left(S_{i}^{p}, S_{i}^{q}\right)$ : purchase price of spinning reserve at bus $\mathrm{i}$,

$T C_{k}\left(T_{k}{ }^{\text {max }}\right)$ : capital cost of capacity for transmission line $\mathrm{k}$.

All decision variables, except those involving reactive power, are nonnegative and the objective function is assumed to have continuous second partial derivatives. For optimal operating and pricing strategies, this objective function is maximized subject to the operating and network constraints inherent in the system. These constraints are described in the following subsections.

\subsubsection{Constraints}

The constraints of the long-run problem are the same as those of the short-run problem and are summarized below. Usually in solving such long-term, large-scale problems, most of these constraints are either simplified or ignored. The constraints are as follows:

(i) Power Flow Equations

The power flow equations, based on Kirchhoff's laws that govern the flow of real and reactive power, for bus $i$ at time $t$ are:

$$
\begin{aligned}
& P_{i t}^{g}+P_{i t}^{s}+P_{i t}^{u}-P_{i t}^{d} \\
& -\sum_{j}\left|V_{i t}\right|\left|V_{j t}\right|\left|V_{i j}\right| \operatorname{Cos}\left(\theta_{i j}+\delta_{j t}-\delta_{i t}\right)=0 \\
& Q_{i t}^{g}+Q_{i t}^{s}+Q_{i t}^{u}-Q_{i t}^{d} \\
& +\sum_{j}\left|V_{i t}\right|\left|V_{j t}\right|\left|V_{i j}\right| \operatorname{Sin}\left(\theta_{i j}+\delta_{j t}-\delta_{i t}\right)=0
\end{aligned}
$$

(ii) Generation Limits

The generating unit capacity constraints may be represented as:

$$
\begin{gathered}
0 \leq P_{i t}^{g} \leq K_{i}^{p} a_{i t} \\
-K_{i}^{q} a_{i t} \leq Q_{i t}^{g} \leq K_{i}^{q} a_{i t}
\end{gathered}
$$

(iii) Transmission Limits

The transmission capacity constraints between buses i and $\mathrm{j}$ are modeled as limits on the volt-ampere flows, namely:

$$
\left|T_{i j t}\right| \leq T_{i j}^{\max } b_{i j t}
$$

(iv) Voltage Limits

The voltage limits can be expressed by the following constraint: 


$$
V_{i}^{\mathrm{min}} \leq\left|V_{i t}\right| \leq V_{i}^{\mathrm{max}}
$$

(v) Security Constraint

The system operational limits boundary imposed by the system security conditions and the voltage collapse constraints can be written as:

$$
g_{t}\left(P_{t}^{d}, Q_{t}^{d}, P_{t}^{g}, Q_{t}^{g}, P_{t}^{s}, Q_{t}^{s}\right) \leq 0
$$

\section{(vi) Spinning Reserve Constraint}

The constraints on the amount of emergency purchase of real and reactive power at bus $i$ at time $t$ are given by:

$$
\begin{aligned}
& 0 \leq P_{i t}^{s} \leq S_{i}^{p} \\
& 0 \leq Q_{i t}^{s} \leq S_{i}^{q}
\end{aligned}
$$

\section{(vii) Emissions Constraints}

The unit specific maximum hourly emissions rate constraints at each generating unit for each pollutant $\mathrm{n} \in \mathrm{NH}$ may be represented as:

$$
e_{i}^{n} P_{i t}^{g} \leq E_{i t}^{n}+A_{i t}^{n}
$$

System-wide emissions constraints designed to limit total annual emissions over the service territory, $\forall \mathrm{n} \in \mathrm{NA}$, may be represented as:

$$
\sum_{i} e_{i}^{n} P_{i t}^{g} \leq E_{t}^{n}+A_{t}^{n}
$$

(viii) Distortion Constraints

In the fixed injection model, we have:

$$
\mathrm{I}_{\mathrm{it}}^{\mathrm{h}}=d_{\mathrm{i}}^{\mathrm{h}} P_{\mathrm{it}}^{\mathrm{d}} \quad \mathrm{h}=2,3,4, \cdots
$$

The harmonic voltages at each bus are given by:

$$
V_{\mathrm{it}}^{\mathrm{h}}=\sum_{j} Z_{\mathrm{ij}}^{\mathrm{h}} I_{\mathrm{jt}}^{\mathrm{h}} \quad \mathrm{h}=2,3,4, \ldots
$$

The total harmonic distortion, THD, can be calculated as:

$$
T H D_{t}=\sum_{i} \frac{\sum_{h=2}^{\infty}\left|V_{\mathrm{it}}{ }^{\mathrm{h}}\right|^{2}}{N B\left|V_{\mathrm{it}}{ }^{1}\right|^{2}}
$$

The constraint on the amount of distortion allowed is

$$
T H D_{t} \leq D_{\max }
$$

(viii) Non-negative constraints on unserved energy

$$
0 \leq P_{i t}^{u}
$$

$$
0 \leq Q_{i t}^{u}
$$

The objective function and all constraints are assumed to have continuous second partial derivatives. The dimension of the problem can be reduced by making the following substitution among the above equations. Equations (6) and (7) represent sets of simultaneous equations that can be solved to determine the voltage and phase angle at each bus as functions of the decision variables.

In practice, a much simple model is used for the purposes of optimal resource planning. There are many different time-periods in the model which are not explicitly shown for simplicity, e.g. operating-1ife of generating units, transmission lines or other capital stock. After initial screening, the planner usually reduces the number of options of generating units and transmission lines for optimization to only a handful of discrete choices. A dynamic programming simulation is then done to determine an optimal plan. However, in the analysis to follow, it is assumed that generating units and transmission lines of a variety of sizes are available. The introduction of economies of scale in capital costs further complicates the task of finding an optimal solution to the resource planning problem.

An important point to note is the absence of an explicit reliability constraint. Conventional resource planning models include a reliability constraint such as a minimum reserve margin or maximum loss of load probability (LOLP) or maximum expected unserved energy (EUE). In the RDP mode1 [9]-[11], the implicit optimal reliability level depends on the relative costs and benefits that customers receive from that level of reliability. In particular, the customer outage costs reflect the customers' willingness-topay for added levels of reliability.

In the model, the discount factor for future costs is not explicitly modeled. This is done in order to keep the notations in the model simple. Discounting to yield a more conventional calculation of the present value of costs can be introduced into the model without changing the formulation in any qualitative sense.

\section{Optimal Investment Planning Strategy}

A significant advance in the theory of investment planning is made possible by the reliability differentiated pricing model. It allows for the integration of the optimal investment strategy across the entire portfolio of technologies used in bulk power supply, from the generator to the point of delivery to the distribution system. Heretofore no theoretical basis for integrated resource planning of such scope has been available.

The benefits of new investment in the model (5) - (22) stem from either the direct reduction in customer outage costs and operational costs, or the indirect reduction of those costs through the relaxation of some constraint or constraints of the model. Investment in new plant and equipment, whether it is in new generating capacity, new 
sources of reactive power, new spinning reserves, new transmission lines, new switchgears, new relaying or other protective devices, new emissions controls, or new power line conditioning equipment, is designed to relieve one or more of the constraints on operations that are a part of the model thus reducing customer outage costs or costs of operation, or directly reducing the costs of operation.

A deficiency of previous models for planning utility bulk power system investments is that the models have not been able to quantify the reliability benefits that investment opportunities in bulk power system improvements afford. Outage costs, however, are an integral part of the reliability differentiated pricing model's formulation. To the extent that a new investment affects a system's reliability, it will change the expected outage cost or the dispatch of real and reactive power in the system or both. The welfare gains associated with higher reliability are thus an integral and natural part of the investment planning evaluation when the reliability differentiated pricing model is used to measure the benefits of an investment decision. The long-run model given by Equations (5) - (22) has three time frames in which decisions are made. The time frames are as follows:

(i) Short-Term: Decisions concerning optimal power dispatch, rationing, and emergency power purchase are all made in this time frame. In the short-term, the capital stock, e.g. generation and transmission capacity, and available spinning reserve are treated as fixed quantities.

(ii) Medium-Term: Decisions concerning spinning reserve purchase, conditional upon given generating and transmission capacities, are made in this time frame. For simplicity, in the long-run models, decisions in this time frame are considered to be long-term decisions.

(iii) Long-Term: Decisions concerning levels of capital stock, e.g. generation and transmission capacities, are made in this time frame. In making decisions in this time frame, it is assumed that optimal short-term and medium-term decisions are made during the operating life of the capital stock.

\subsection{Criterion for Optimal Investment Decisions}

The long-term investment decisions on generation and transmission capacity levels are optimal when the following first order conditions for the long-run model (5)-(22) are satisfied, assuming that optimal short-term and medium-term decisions are made as:

$$
\begin{array}{ll}
\frac{\partial L}{\partial K_{i}^{p}}=0 & \forall i \\
\frac{\partial L}{\partial K_{i}^{q}}=0 & \forall i \\
\frac{\partial L^{\text {max }}}{\partial T_{k}^{\text {a }}}=0 & \forall k
\end{array}
$$

where the simplifying assumption is that real and reactive power generation capacities can be separately considered and that the Lagrangian (L) of the problem in Eq. (5) is differentiable with respect to these variables. One simple way to overcome the first problem is to combine the real and reactive power components to have capacities specified in volt-ampere (MVA) terms, as used in defining transmission constraints. These conditions are analyzed and discussed in the following subsections for investments in generation and transmission capacity addition.

\subsection{Optimal Conditions for Generation Investment Decisions}

Consider the long-run resource planning problem (5) (22) of the welfare maximizing utility. Assuming that the short-term and medium-term decisions are optimally made, the long-run model reduces to one of determining the optimal levels of capital stock and, in particular, all other variables and the objective function may be considered as functions of the long-term variables. Thus conditions (23) and (24) give:

$$
\begin{aligned}
& \frac{\partial T C_{k}}{\partial T_{k}^{\max }}=-\sum_{t \in T} \sum_{j}\left\{\frac{\partial O C_{j t}}{\partial T_{k}^{\max }}+\frac{\partial P C_{j t}}{\partial T_{k}^{\max }}+\frac{\partial E C_{j t}}{\partial T_{k}^{\max }}\right\} \\
& \frac{\partial C C_{i}}{\partial K_{i}^{q}}=-\sum_{t \in T} \sum_{j}\left\{\frac{\partial O C_{j t}}{\partial K_{i}^{q}}+\frac{\partial P C_{j t}}{\partial K_{i}^{q}}+\frac{\partial E C_{j t}}{\partial K_{i}^{q}}\right\}
\end{aligned}
$$

where $\mathrm{T}$ represents the operating life of the generating unit.

Equations (26) and (27) state that the optimal investment (capacity) level of generator $i$ should be such that the costs of an additional incremental investment equals the present value of the expected benefits from it over the operating life of the generator. The benefits are: (i) Reduction in customer outage costs due to increased available capacity; (ii) Reduction in total production cost due to operating efficiency gains; and (iii) Reduction in emergency power purchase costs, again, due to increased system capacity.

\subsection{Optimal Condition for Transmission Investment Decisions}

Consider again the long-run resource planning problem (5) - (22) of the welfare maximizing utility. Assuming that the short-term and medium-term decisions are optimally made, the long-run model reduces to one of determining the optimal levels of capital stock and, in particular, all other variables and the objective function may be considered as functions of the long-term variables. Thus condition (25) gives:

$$
\frac{\partial T C_{k}}{\partial T_{k}^{\max }}=-\sum_{t \in T} \sum_{j}\left\{\frac{\partial O C_{j t}}{\partial T_{k}^{\max }}+\frac{\partial P C_{j t}}{\partial T_{k}^{\max }}+\frac{\partial E C_{j t}}{\partial T_{k}^{\max }}\right\}
$$

where $\mathrm{T}$ represents the operating life of the transmission line. 
Eq. (28) states that the optimal capacity investment level of transmission line $\mathrm{k}$ should be such that the costs of an additional incremental investment in capacity equals the present value of the expected benefits from it over the operating life of the transmission line. The benefits are: (i) Reduction $m$ customer outage costs due to increased reliability and security of the system; (ii) Reduction in total production cost due to more efficient delivery of electricity; and (iii) Reduction in emergency power purchase costs due to increased system reliability and power transportation capability.

\section{Case Study}

The case study determines the socially "optimal" generation expansion plan for a large utility in Texas based on information about customer outage costs and costs of capacity additions. The Electric Generation Expansion Analysis System (EGEAS) was used to determine the optimal expansion plans, since it is basically designed to perform optimal generation expansion planning. The results of the study indicate that, given the customer outage cost information of the utility, fuel price forecasts, and other assumed costs and escalation rates, the socially optimal expansion plan has substantially lower investment in new generating capacity as compared to the utility's own expansion plan, i.e. the reserve margin from a societal welfare point of view is much lower than the minimum reserve margin criterion used by the utility in developing its expansion plan.

\subsection{Reserve Margin in Conventional Resource Planning}

The reserve margin is defined here as the percentage by which the installed capacity exceeds system peak load for a particular year. Utilities usually use a certain minimum reserve margin percentage, which the system generating capacity is required to meet, in planning their capacity expansion. This minimum reserve margin is, in general, obtained from some engineering practice or rule-of-thumb, for example, the Electric Reliability Council of Texas (ERCOT) specification of $15 \%$ minimum reserve margin, which may not be socially optimal. Thus, in the test case, any assumption of minimum reserve margin for capacity expansion planning of the utility is discarded, and an optimal reserve margin, from a. societal cost point of view, is determined. This is based on customer outage costs, since outage costs reflect the price customers are willing to pay to avoid an outage. Outage costs thus provide an accurate measure of the value of reliability to the customers. Table 4.1 gives the estimated system outage costs for the utility under consideration.

The expected unserved energy (EUE), which gives the expected customer outage or interruption costs when a cost of unserved energy is associated with it, decreases as the system reserve margin is increased. This is due to the fact
Table 4.1. Estimated System Outage Cost

\begin{tabular}{c|c|c|c}
\multicolumn{4}{c}{$\begin{array}{c}\text { Estimated High System Outage Cost } \\
(1-4 \text { hour outages })\end{array}$} \\
\hline Class & $\begin{array}{c}\text { Utility En- } \\
\text { ergy Mix }(\%)\end{array}$ & $\begin{array}{c}\text { Class outage } \\
\text { Cost }(\$ / \mathrm{MWH})\end{array}$ & $\begin{array}{c}\text { System Outage } \\
\text { Cost (\$/MWH) }\end{array}$ \\
\hline Residential & 28.6 & 2,970 & 850 \\
\hline Commercial & 22.0 & 16,380 & 3,600 \\
\hline Industrial & 49.4 & 12,080 & 5,970 \\
\hline System Average & & & 10,420 \\
\hline
\end{tabular}

Estimated Average System Outage Cost (1-4 hour outages)

\begin{tabular}{c|c|c|c}
\hline Class & $\begin{array}{c}\text { Utility Energy } \\
\text { Mix }(\%)\end{array}$ & $\begin{array}{c}\text { Class outage } \\
\text { Cost }(\$ / \mathrm{MWH})\end{array}$ & $\begin{array}{c}\text { System Outage } \\
\text { Cost }(\$ / \mathrm{MWH})\end{array}$ \\
\hline Residential & 28.6 & 1,770 & 510 \\
\hline Commercial & 22.0 & 11,280 & 2,480 \\
\hline Industrial & 49.4 & 8,100 & 4,000 \\
\hline System Average & & & 6,990 \\
\hline
\end{tabular}

Estimated Low System Outage Cost (1-4 hour outages)

\begin{tabular}{c|c|c|c}
\hline Class & $\begin{array}{c}\text { Utility Energy } \\
\text { Mix }(\%)\end{array}$ & $\begin{array}{c}\text { Class outage } \\
\text { Cost }(\$ / \mathrm{MWH})\end{array}$ & $\begin{array}{c}\text { System Outage } \\
\text { Cost }(\$ / \mathrm{MWH})\end{array}$ \\
\hline Residential & 28.6 & 400 & 110 \\
\hline Commercial & 22.0 & 5,550 & 1,220 \\
\hline Industrial & 49.4 & 6,140 & 3,030 \\
\hline System Average & & & 4,370 \\
\hline
\end{tabular}

Source: HL\&P NOI 90-A-104

that with higher reserve margins, (that is, with greater installed generating capacity), the expected load that the utility is unable to supply because of forced outages of units and thus capacity shortage is lower than with lesser installed generating capacity.

\subsection{Implementation Using EGEAS}

EGEAS uses a probabilistic production costing method based on the Booth-Balleriaux algorithm. This method is capable of handling a wide variety of generating units and can model partial outage states, automatic maintenance scheduling, must-run units, and spinning reserves. In the Booth-Balleriaux algorithm, system load is represented by a load duration curve (LDC). The expected unserved energy (EUE) at any time period is found by a convolution process involving the corresponding LDC for that period. In this process, after a unit is loaded, its forced outages are convolved onto the current equivalent load duration curve (ELDC) to derive the new ELDC, which reflects the demand seen by the next unit in the merit order. The height of the ELDC at the unit's capacity point gives the probability that the system demand exceeds the available capacity. After the last unit is loaded, this height gives the probability that the entire system load cannot be met, i.e., loss of load probability (LOLP), and the area under the ELDC beyond the capacity point of a unit, when multiplied by the number of hours in the time period, gives the expected unserved energy (EUE). 
The objective function for generation expansion optimization in EGEAS is the total present worth of revenue requirements. It includes the fixed capital charges for new generating units and the operating costs associated with generation to meet the system load. These operating costs include fuel costs, fixed and variable components of O\&M costs, purchased energy costs, and unserved energy costs. Thus, EGEAS already has the cost of EUE included in the objective function, as is required for the analysis to be performed. Thus the objective function of the generation expansion optimization model is the same as the total cost. The only problem is that only one value of system unserved energy cost (\$/MWH) can be specified in the model. Table 3.2 lists the cost of unserved energy or customer cost for some emergency operating procedures that are available to the utility. It is observed that a considerable amount of demand exists that can be curtailed in the event of an emergency at a cost of $\$ 200 / \mathrm{MWH}$ or less. Thus, an unserved energy cost of $\$ 200 / \mathrm{MWH}$ is used in the analysis as the system unserved energy cost for the test case.

The model was executed with no reliability constraints, such as minimum and maximum reserve margins, maximum LOLP, or maximum EUE, and for system unserved energy costs in the range of $\$ 10 / \mathrm{MWH}$ to $\$ 2000 / \mathrm{MWH}$ with an escalation rate of $9 \%$ annually.

Table 4.2. Customer Costs for Emergency Operating Procedures

\begin{tabular}{c|c|c|c}
\hline $\begin{array}{c}\text { Emergency } \\
\text { Operating } \\
\text { Procedure }\end{array}$ & $\begin{array}{c}\text { Capacity } \\
(\mathrm{MW})\end{array}$ & $\begin{array}{c}\text { Availability } \\
(\%)\end{array}$ & $\begin{array}{c}\text { Customer } \\
\text { Cost } \\
(\$ / \mathrm{MWH})\end{array}$ \\
\hline $\begin{array}{c}\text { Emergency } \\
\text { Purchases } \\
\text { from ERCOT }\end{array}$ & 1500 & $\begin{array}{c}\text { Varies by } \\
\text { season }\end{array}$ & $(\mathrm{GT}+10 \%)$ \\
\hline $\begin{array}{c}\text { Interruptible } \\
\text { Demand }\end{array}$ & $\begin{array}{c}(95 \% \text { of inter. } \\
\text { forecast })\end{array}$ & 100 & 0 \\
\hline $\begin{array}{c}\text { Voltage } \\
\text { Reduction }\end{array}$ & $\begin{array}{c}(3.5 \% \text { of firm } \\
\text { demand })\end{array}$ & 100 & 200 \\
\hline $\begin{array}{c}\text { Voluntary } \\
\text { Industrial } \\
\text { Reduction }\end{array}$ & $\begin{array}{c}(3 \% \text { of ind. } \\
\text { demand })\end{array}$ & 100 & 100 \\
\hline $\begin{array}{c}\text { Media Appeal } \\
\text { for Reduction }\end{array}$ & $\begin{array}{c}(1 \% \text { of firm } \\
\text { demand })\end{array}$ & 50 & 6,990 \\
\hline $\begin{array}{c}\text { Involuntary } \\
\text { Reduction }\end{array}$ & $\begin{array}{c}\text { (Total System } \\
\text { Demand })\end{array}$ & 100 & 200 \\
\hline
\end{tabular}

Source: HL\&P NOI90-A-104

Table 4.3. Fuel Price Forecast for Test Case

FUEL PRICE FORECASTS

Dollars per MMBtu

\begin{tabular}{c|c|c|c|c|c}
\hline Year & \multicolumn{2}{|c|}{ Natural Gas } & Lignite & Coal & Nuclear \\
\hline & Base & High & & & \\
\hline 2011 & 1.63 & 1.63 & 1.62 & 2.29 & 0.60 \\
\hline 2012 & 1.89 & 1.89 & 1.63 & 2.36 & 0.61 \\
\hline 2013 & 1.97 & 2.04 & 1.80 & 2.44 & 0.61 \\
\hline 2014 & 2.07 & 2.22 & 1.92 & 2.51 & 0.60 \\
\hline 2015 & 2.17 & 2.40 & 2.06 & 2.59 & 0.60 \\
\hline 2016 & 2.24 & 2.57 & 2.30 & 2.67 & 0.60 \\
\hline 2017 & 2.35 & 2.79 & 2.52 & 2.74 & 0.61 \\
\hline 2018 & 2.47 & 3.04 & 2.72 & 2.91 & 0.62 \\
\hline 2019 & 2.59 & 3.30 & 2.87 & 3.07 & 0.65 \\
\hline 2020 & 2.73 & 3.59 & 3.08 & 3.23 & 0.75 \\
\hline
\end{tabular}

Since optimal expansion plans are greatly dependent on projected fuel price and since natural gas price forecasts can vary considerably, they are evaluated for two different cases: (a) Base Case, where natural gas prices are forecasted to escalate at a relatively low rate; and (b) High Case, where natural gas prices are forecasted to escalate at a relatively high rate. The fuel price forecasts-used in the analysis are given in Table 4.3.

\subsection{Case Study Results}

The optimal expansion plans Base Natural Gas Price Case and the Company Plan is shown in Table 4.4. Plan 3 is the test case previously discussed, since in this case the cost of unserved energy is $\$ 200 / \mathrm{MWH}$ and, as shown in Table 4.2, a considerable amount of demand exists that can be curtailed at this cost. The comparison of Plan 3 (Base Natural Gas Price Case) and the Company Plan shows that the minimum reserve margin for Plan 3 is $10 \%$, while that of the Company Plan is about $17 \%$, a difference of about $7 \%$. The capacity additions in the Company Plan are: $400 \mathrm{MW}$ of firm capacity purchased in 2015 , two $110 \mathrm{MW}$ gas turbines and $158 \mathrm{MW}$ cogeneration capacity purchased in 2016, two $110 \mathrm{MW}$ gas turbines in 2018 , and a $645 \mathrm{MW}$ lignite unit in 2020 . On the other hand, the capacity additions in Plan 3 (Base Natural Gas Price Case) are: 158MW cogeneration capacity purchased in 2015 , two $110 \mathrm{MW}$ gas turbines in 2018, and 110MW gas turbines in 2020. This lesser capacity addition in Plan 3 as compared to the Company Plan, of course, gives rise to higher values of EUE, but results in lower levels of revenue requirement, and hence, lower average rates.

Table 4.4. Comparison of Plan 3 and Company Plan

\begin{tabular}{c|c|c|c|c|c|c}
\hline \multirow{2}{*}{ Year } & \multicolumn{2}{|c|}{ Capacity Addition } & \multicolumn{2}{c|}{ Reserve Margin } & \multicolumn{2}{c}{ EUE } \\
\cline { 2 - 7 } & Company & Plan 3 & $\begin{array}{c}\text { Comp. } \\
(\%)\end{array}$ & $\begin{array}{c}\text { Plan 3 } \\
(\%)\end{array}$ & $\begin{array}{c}\text { Comp. } \\
(\mathrm{GWH})\end{array}$ & $\begin{array}{c}\text { Plan 3 } \\
(\mathrm{GWH})\end{array}$ \\
\hline 2011 & & & 26 & 26 & 10 & 10 \\
\hline 2012 & & & 24 & 24 & 7 & 7 \\
\hline 2013 & & 22 & 22 & 8 & 8 \\
\hline 2014 & & 20 & 20 & 7 & 7 \\
\hline 2015 & $400 M W$ Firm & $\begin{array}{c}158 \mathrm{MW} \\
\text { Cogen }\end{array}$ & 21 & 19 & 18 & 28 \\
\hline 2016 & $\begin{array}{c}2 \times 110 M W ~ G T \\
158 M W C o g e n\end{array}$ & & 19 & 14 & 7 & 26 \\
\hline 2017 & & 19 & 14 & 11 & 38 \\
\hline 2018 & $2 \times 110 M W$ GT & $\begin{array}{c}2 \times 110 M \\
\text { WGT }\end{array}$ & 19 & 14 & 13 & 52 \\
\hline 2019 & & & 17 & 12 & 8 & 35 \\
\hline 2020 & $645 M W$ LIG & $\begin{array}{c}110 M W \\
\text { GT }\end{array}$ & 19 & 10 & 5 & 49 \\
\hline
\end{tabular}

\section{Conclusion and Future Studies}

The suggestion that consumers of electricity be given the choice of opting for different levels of service reliability has often been made. Customers would then subscribe to the level of reliability that best suits them arid their processes. It is argued that such a choice would unbundle ser- 
vices and thus result in improved efficiency and benefits to both consumers and producers. But priority pricing schemes developed thus far introduce economic inefficiencies and fall short of attaining welfare maximization.

Real-time pricing or spot market pricing schemes, on the other hand, fail to take into account the long-run costs of producing electricity such as capital costs of capacity addition, and, in real systems in-general, are unable to recover sufficient revenue in order to meet the utility's revenue requirement. Schemes to recover the required revenue suggested so far, e.g. Ramsey-type pricing schemes applied to real-time pricing, result in loss of welfare. The pricing policy proposed in this paper overcomes most of these shortcomings of both priority pricing and real-time pricing schemes, and, under certain assumptions, is able to recover revenue without any loss in welfare as well as differentiate prices for differentiated levels of service reliability based on consumer outage costs.

We propose a Reliability Differentiated Pricing for a long-run system expansion planning based on outage costs, and presents the mathematical conditions for optimal investment decisions. Future studies might deal with the implications of the long-run model to pricing firm capacity, transmission services, and wheeling services, and numerical case studies.

\section{Acknowledgements}

This work has been supported by KEPCO (R-200702210 : Development of Electric Power Market Simulator), which is funded by MKE (Ministry of Knowledge \& Economy)

\section{References}

[1] M. Munasinghe and A. Sanghvi, "Reliability of Electricity Supply, Outage Costs and Value of Service: An Overview," The Energy Journal, Vol.9, pp.1-18, 1988.

[2] A. Sanghvi, "Economic Costs of Electricity Supply Interruptions: US and Foreign Experience," Energy Economics 4 (3), pp.180-198, 1982.
[3] A. Sanghvi, "Household Welfare Loss Due to Electricity Supply Disruptions," The Energy Journal, Vol.4, Special Electricity Issue, pp.33-54, 1983.

[4] S. N. Siddiqi and M. L. Baughman, "Reliability Differentiated Real-Time Pricing of Electricity," Paper 92-WM-115-6, IEEE 1992 Winter Power Meeting, New York, NY, January 26-30, 1992.

[5] S. N. Siddiqi and M. L. Baughman, "Optimal Pricing of Non-Utility Generated Electric Power," Paper 93WM-149-5, IEEE 1993 Winter Power Meeting, Columbus, OH, January 31 - February 5, 1993.

[6] W. Vickery, "Responsive Pricing and Public Utilities," Bell Journal of Economics and Management Sciences, Vol.2, No.1, 1971.

[7] C. K. Woo and N. Toyama, "Service Reliability and the Optimal Interruptible Rate Option in Residential Electricity Pricing," Energy Journal, 7:3, 1986.

[8] C. K. Woo and K. Train, "The Cost of Electric Power Interruptions to Commercial Firms," The Energy Journal, Vol.9, pp. 161-172, 1988.

[9] D. W. Caves, J. A. Herriges, and R. J. Windle, "Customer Demand for Service Reliability, A Synthesis of the Outage Costs Literature," EPRI Report P-2801, September 1989.

[10] H. P. Chao, S. Oren, S. Smith, and R. Wilson, "Service Design in Electric Power Industry," Report P6543, Electrical Power Research Institute, 1990.

[11] H. P. Chao and R. Wilson, "Priority Service: Pricing, Investment, and Market Organization," American Economic Review, 77: 899-916, 1987.

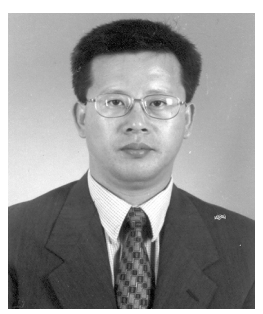

Balho H. Kim He was born in BongHwa, Korea. He received his BSEE from Seoul National University, and his MSEE and Ph.D from the University of Texas at Austin. Currently, he is a professor in the School of Electrical Engineering at Hongik University. His research fields include Optimal Power Flow, Public Utility Pricing, Electricity Market Design and Operation, Resource Planning, and Demand Management. 